\title{
Impact of KCNQ1, CDKN2A/2B, CDKAL1, HHEX, MTNR1B, SLC3OA8, TCF7L2, and UBE2E2 on risk of developing type 2 diabetes in Thai population
}

Nattachet Plengvidhya ${ }^{1}$, Chutima Chanprasert ${ }^{1,2}$, Nalinee Chongjaroen ${ }^{3}$, Pa-thai Yenchitsomanus ${ }^{4}$, Mayuree Homsanit ${ }^{5}$ and Watip Tangjittipokin ${ }^{3^{*}}$ (D)

\begin{abstract}
Background: Several type 2 diabetes (T2D) susceptibility loci identified via genome-wide association studies were found to be replicated among various populations. However, the influence of these loci on T2D in Thai population is unknown. The aim of this study was to investigate the influence of eight single nucleotide polymorphisms (SNPs) reported in GWA studies on T2D and related quantitative traits in Thai population.
\end{abstract}

Methods: Eight SNPs in or near the KCNQ1, CDKN2A/2B, SLC3OA8, HHEX, CDKAL1, TCF7L2, MTNR1B, and UBE2E2 genes were genotyped. A case-control association study comprising 500 Thai patients with T2D and 500 ethnicallymatched control subjects was conducted. Associations between SNPs and T2D were examined by logistic regression analysis. The impact of these SNPs on quantitative traits was examined by linear regression among case and control subjects.

Results: Five SNPs in KCNQ1 (rs2237892), CDK2A/2B (rs108116610, SLC30A8 (rs13266634), TCF7L2 (rs7903146) and MTNR1B (rs1387153) were found to be marginally associated with risk of developing T2D, with odds ratios ranging from 1.43 to 2.02 ( $p=0.047$ to $3.0 \times 10-4)$ with adjustments for age, sex, and body mass index. Interestingly, SNP rs13266634 of SLC30A8 gene reached statistical significance after correcting for multiple testing $(p=0.0003)(p<0$. 006 after Bonferroni correction). However, no significant association was detected between HHEX (rs1111875), CDKAL1 (rs7756992), or UBE2E2 (rs7612463) and T2D. We also observed association between rs10811661 and both waist circumference and waist-hip ratio ( $p=0.007$ and $p=0.023$, respectively). In addition, rs 13266634 in SLC30A8 was associated with glycated hemoglobin $(p=0.018)$, and rs7903146 in TCF7L2 was associated with high-density lipoprotein cholesterol level $(p=0.023)$.

Conclusion: Of the eight genes included in our analysis, significant association was observed between KCNQ1, CDKN2A/2B, SLC3OA8, TCF7L2, and MTNR1B loci and T2D in our Thai study population. Of these, CDKN2A/2B, SLC30A8, and TCF7L2 genes were also significantly associated with anthropometric, glycemic and lipid characteristics. Larger cohort studies and meta-analyses are needed to further confirm the effect of these variants in Thai population.

Keywords: Type 2 diabetes, Association study, Single nucleotide polymorphisms, Thai population, Genome-wide association study

\footnotetext{
* Correspondence: watip.tan@mahidol.edu

${ }^{3}$ Department of Immunology, Faculty of Medicine Siriraj Hospital, Mahidol

University, Bangkok, Thailand

Full list of author information is available at the end of the article
}

(c) The Author(s). 2018 Open Access This article is distributed under the terms of the Creative Commons Attribution 4.0 International License (http://creativecommons.org/licenses/by/4.0/), which permits unrestricted use, distribution, and reproduction in any medium, provided you give appropriate credit to the original author(s) and the source, provide a link to the Creative Commons license, and indicate if changes were made. The Creative Commons Public Domain Dedication waiver (http://creativecommons.org/publicdomain/zero/1.0/) applies to the data made available in this article, unless otherwise stated. 


\section{Background}

The prevalence of diabetes, especially type 2 diabetes (T2D), continues to increase worldwide. The International Diabetes Federation (IDF) has estimated that the prevalence of diabetes will increase from 451 million in 2017 to 693 million by 2045 [1]. The number of people with diabetes will increase as a result of changes in lifestyle caused by urbanization, high population growth, ageing population, and high economic growth. In 2017, the prevalence of diabetes among adults in Thailand was estimated to be $8.3 \%$ [1]. T2D is a complex and multifactorial disease that is characterized by impaired insulin secretion and insulin resistance [2, 3]. T2D is associated with significant morbidity and mortality, especially decreased life expectancy and reduced quality of life, as well as high cost of treatment and reduced productivity. Although the etiology of T2D is not thoroughly understood, genetic and environmental factors are thought to be involved in the pathogenesis of this disease.

Prior to the development of currently available methods and technologies, linkage and candidate gene association studies were conducted; however, these two methods could identify only a few genes involved in the pathogenesis of T2D (i.e., PPARG, KCNJ11, CAPN10, and TCF7L2). Advances in genotyping technology and genetic information have facilitated the application of genome-wide association (GWA) studies to identify T2D susceptibility genes. Currently, over 120 common risk variants have been successfully identified as being associated with T2D using this approach [4-12]. Several reproducible GWA studies confirmed these well-established susceptibility genes, and they were found to be replicated for association with T2D and diabetes-related traits in or near SLC30A8, KCNQ1, CDC123, HNF1B, KCNJ11, TCF7L2, CDKAL1, CDKN2A/2B, PPARG, HHEX, IGF2BP2, GLIS3, JAZF1, WFS1, and MTNR1B in Europeans and East Asians. Most of the susceptibility risk loci identified were shared among East Asians and populations with Europeans ancestry. However, significant association signals at these shared loci seems to be independent among populations. This suggests that the pathogenesis of the disease is common among populations, but that risk variants are often population specific. These differences in risk variants between Europeans and East Asians are likely due to differences in genetic background, risk allele frequencies, and characteristics, such as body shape, food and drink, culture, and other lifestyle factors. For example, association of variants within KCNQ1 $[6,13]$ was primarily reported in GWA studies in East Asians; however, these variants were not identified in any of the larger GWA studies in European populations, because there are different frequencies of these variants between East Asian and European populations. Similarly, variants within UBE2E2 and $C 2 C D 4 A-C 2 C D 4 B$ were reported to be associated with T2D by two relatively small East Asian GWA studies, while these loci did not reach genome-wide significance in European studies $[4,5,14]$. Furthermore, there are relatively large frequency differences between population groups for rs7903146 within TCF7L2 [15]. Although numerous T2D risk loci were identified from GWA studies in multi-ethnic populations, the functional impact of these risk loci still needs to be elucidated. Most of these risk loci likely affect insulin secretion and beta-cell function, with a few potentially involved in insulin action. Given this variability among populations, it is important that we understand the association between genetic variations and T2D, and the roles of these T2D risk loci in Thai population. In this study, we investigated single nucleotide polymorphisms (SNPs) that were previously found to be associated with T2D in Asian populations. We focused on the following eight SNPs: 1) potassium voltage-gated channel, KQT-like subfamily, member 1 (KCNQ1; rs2237892); 2) a variant found near cyclin-dependent kinase inhibitor 2A (CDKN2A/2B; rs10811661); 3) a zinc transporter member of solute carrier family 30 (SLC30A8; rs13266634); 4) hematopoietically-expressed homeobox (HHEX; rs1111875); 5) CDK5 regulatory subunit-associated protein 1-like 1 (CDKAL1; rs7756992); 6) melatonin receptor type 1B (MTNR1B; rs1387153); 7) transcription factor-7-like 2 (TCF7L2; rs7903146); and, 8) Ubiquitin Conjugating Enzyme E2 E2 (UBE2E2; rs7612463). Our specific aim was to investigate the influence of these eight SNPs on T2D and related quantitative traits in Thai population.

\section{Methods}

\section{Study subjects}

A total of 1000 individuals, including 500 Thai T2D patients and 500 ethnically-matched control subjects were enrolled during the 2005 to 2015 study period. T2D patients were recruited at the Siriraj Diabetes Center, Department of Endocrinology and Metabolism, Faculty of Medicine Siriraj Hospital, Mahidol University, Bangkok, Thailand. Siriraj Hospital is Thailand's largest national tertiary referral center. Diabetes was diagnosed based on American Diabetes Association (ADA) criteria [3]. Control subjects consisted of 500 individuals without diabetes who were enrolled from the Health Check-up Center of the Department of Preventive and Social Medicine, Faculty of Medicine Siriraj Hospital, Mahidol University. Healthy controls had to satisfy all of the following inclusion criteria: age $>40$ years, no family history of diabetes in any first-degree relatives, fasting plasma glucose $<100 \mathrm{mg} / \mathrm{dl}(6.1 \mathrm{mmol} / \mathrm{l})$, and $\mathrm{HbA}_{1 \mathrm{c}}$ $<5.7 \%$. Written informed consent was obtained from all participants after a full explanation of the study's purpose and protocol. The study protocol and informed 
consent procedures were approved by the Siriraj Institutional Review Board (SIRB), Faculty of Medicine Siriraj Hospital, Mahidol University, Bangkok, Thailand (COA no. Si491/2014). This study was conducted in accordance with the principles set forth in the Declaration of Helsinki and all of its subsequent amendments.

\section{SNP selection and genotyping}

Genomic DNA was extracted from peripheral blood leukocytes using the standard phenol-chloroform method. Eight common SNPs from eight loci were selected for analysis of association with T2D. First, we selected loci that demonstrated strong association with T2D from prior GWA studies and meta-analyses in Caucasian and Asian populations (Chinese, Japanese, and Korean). The following 8 loci were selected for investigation in this study: KCNQ1, CDKN2A/2B, HHEX, SLC30A8, CDKAL1, UBE2E2, TCF7L2, and MTNR1B. Subsequently, one SNP was selected in each locus according to the following criteria: (a) the most replicated SNP in each locus with the lowest reported $p$-value was selected; and, (b) that SNP as a tagSNP within observed Linkage Disequilibrium (LD) based on HapMap project (CHB data) using Haploview 4.2, except for SNP rs10811661 near CDKN2A/2B. SNP rs10811661 is out of LD mapping; however, this SNP is the most highly replicated T2D in Caucasian and Asian populations. The following $8 \mathrm{SNPs}$ were tested for association with T2D: KCNQ1 (rs2237892), CDKN2A/2B (rs10811661), CDKAL1 (rs7756992), HHEX (rs1111875), MTNR1B (rs1387153), SLC30A8 (rs13266634), TCF7L2 (rs7903146), and UBE2E2 (rs7612463). Genotyping of these SNPs was performed using high-resolution melting (HRM) analysis or polymerase chain reaction-restriction fragment length polymorphism (PCR-RFLP) method (Additional file 1: Tables S2 and S3, respectively). For HRM assay, PCR was performed in 96-well plates with a total volume of $10 \mathrm{ul}$ in each, which consisted of $50 \mathrm{ng}$ of DNA template, $10 \mathrm{uM}$ of forward and reverse primers, $1 \times$ buffer, $1.5 \mathrm{mM}$ of $\mathrm{MgCl}_{2}, 0.5 \mathrm{U}$ of Taq DNA polymerase (Bioline, Inc., London, UK), and $1 \times$ ResoLight dye (Roche Diagnostics, Risch-Rotkreuz, Switzerland). HRM assays were performed using a LightCycler $^{\circ} 480$ System with LightCycler ${ }^{\circledR} 480$ Gene Scanning Software Version 1.5 (Roche Diagnostics). PCR program consisted of an initial denaturation step at $95{ }^{\circ} \mathrm{C}$ for $10 \mathrm{~min}$, followed by a 50-cycle program consisting of denaturation at $95{ }^{\circ} \mathrm{C}$ for $30 \mathrm{~s}$, annealing at the condition appropriate for each SNP (Additional file 1: Table S2) for $30 \mathrm{~s}$, and elongation at $72{ }^{\circ} \mathrm{C}$ for $30 \mathrm{~s}$, with a single acquisition mode for fluorescence signals. The melting program included denaturing at $95{ }^{\circ} \mathrm{C}$ for $30 \mathrm{~s}$, annealing at $40{ }^{\circ} \mathrm{C}$ for $30 \mathrm{~s}$, and subsequent melting that included a continuous fluorescent reading of fluorescence from $60{ }^{\circ} \mathrm{C}$ to $99^{\circ} \mathrm{C}$ at a rate of 25 acquisitions per one degree Celcius. Quality of SNP genotyping was checked by the reproducibility of genotypes of control DNA samples, which included homozygous major allele, heterozygous major and minor alleles, homozygous minor allele, and negative control. The genotyping success rate was greater than $98 \%$ for all SNPs. Genotyping of SLC30A8 (rs13266634) was performed by PCR, followed by restriction enzyme digestion according to the manufacturer's instructions (Fermentas, Vilnius, Lithuania). All genotypes were confirmed by direct sequencing. Primer sequences and conditions of HRM and PCR-RFLP assays are presented in Additional file 1: Tables S2 and S3, respectively.

\section{Statistical analysis}

Hardy-Weinberg equilibrium (HWE) was evaluated using an exact test implemented in the SNPstats online program (http://bioinfo.iconcologia.net/snpstats/start.htm). Analyses to determine associations between SNPs and T2D were performed under the additive, dominant, and recessive models using logistic regression analysis, with/without adjustment for age, sex, and BMI as covariates. In the additive model, homozygous for risk allele (1/1), heterozygotes $(1 / 0)$, and homozygous for non-risk allele $(0 / 0)$ were coded to a continuous variable for the genotype $(2,1$, and 0$)$, respectively. The dominant model was defined as $1 / 1+1 / 0$ vs. $0 / 0$, and the recessive model was defined as $1 / 1$ vs. $1 / 0$ $+0 / 0$. Bonferroni test was used to correction for multiple testing. A $p<0.006$ (0.05 divided by 8 , the total number of SNPs studied) was regarded as being statistically significant. Characteristics were compared and tested for significant differences between patients and controls using Student's t-test or Mann-Whiney $\mathrm{U}$ test for continuous variables, and using $\mathrm{X}^{2}$ test for categorical variables.

Data are reported as mean \pm standard deviation for quantitative variables with normally distributed data, and as median and interquartile range for non-normally distributed data. Linear regression was applied to test quantitative variables for differences between genotype groups by adjusting for age and sex for BMI; for age, sex, and BMI for weight, waist circumference, and waist-to-hip ratio; or, age, sex, and medication (as appropriate) for all other traits. Other adjustments that were made included anti-diabetic medications (e.g., sulphonylurea, metformin, and DPP4 inhibitor) for fasting plasma glucose (FPG) and glycated hemoglobin $\left(\mathrm{HbA}_{1 \mathrm{c}}\right)$; anti-hypertensive medications (e.g., ACE inhibitors, calcium channel blocker, beta blocker) for systolic and diastolic blood pressure; and, anti-hyperlipidemic medications (e.g., statins, fibrate, and ezetimibe) for total cholesterol (TC), triglycerides (TG), low-density lipoprotein (LDL-C), and high-density lipoprotein (HDL-C). Values of FPG, $\mathrm{HbA}_{1 \mathrm{c}}$, TG, LDL-C, and HDL-C were natural logarithm-transformed to normal 
distributions before statistical analysis. Data analysis was performed using SPSS Statistics version 17.0 (SPSS, Inc., Chicago, IL, USA).

The statistical power of this study for each SNP was estimated using Quanto (http://biostats.usc.edu/Quanto.html), and powers were calculated using ORs from previously published studies, sample sizes, and minor allele frequencies (MAF). In the present study, the prevalence of T2D in Thailand was $8.3 \%$ [16], and the type 1 error rate was 0.006 .

\section{Results}

\section{Clinical and biochemical characteristics}

The clinical characteristics of both study groups are presented in Table 1 . There were significant differences between patients and control group individuals relative to age, weight, waist circumference, waist-to-hip ratio (WHR), body mass index (BMI), systolic blood pressure (SBP), diastolic blood pressure (DBP), fasting plasma glucose (FPG), glycated hemoglobin $\left(\mathrm{HbA}_{1 \mathrm{c}}\right)$, total cholesterol (TC), triglycerides (TG), low-density lipoprotein cholesterol (LDL-C), and high-density lipoprotein cholesterol (HDL-C) (all $p<0.05)$.

\section{Analysis of association between SNPs and T2D}

We genotyped eight SNPs in a case-control cohort of 1000 Thai subjects, including $500 \mathrm{~T} 2 \mathrm{D}$ patients and 500 non-diabetic controls. The allele and genotype distributions of SNPs are summarized in Table 2. All 8 SNP genotypes were in Hardy-Weinberg equilibrium in the control group (all $p>0.05$ ) (Additional file 1: Table S1). Logistic regression analysis was used to assess association between each SNP and T2D in 3 different genetic models (dominant, recessive, and additive). Among the 8 evaluated SNPs, the following SNPs were found to be marginally associated with increased risk of T2D after adjustment for age, sex, and BMI: KCNQ1 (rs2237892) [odds ratio (OR): 2.02, 95\% CI: 1.08-3.79; $p=$ 0.020 (additive model)] and [OR: 1.43, 95\% CI: $1.06-1.92 ; p$ $=0.018$ (recessive model)]; $C D K N 2 A / 2 B$ (rs10811661) [OR: 1.65, 95\% CI: $1.01-2.71 ; p=0.044$ (additive model)]; SLC30A8 (rs13266634) [OR: 1.86, 95\% CI: 1.19-2.90; $p=$ 0.006 (additive model)] and [OR: 1.81, 95\% CI: 1.31-2.50; $p$ $=0.0003$ (recessive model)]; TCF7L2 (rs7903146) [OR: 1.70, 95\% CI: $1.06-2.72 ; p=0.025$ (dominant model)]; and, MTNR1B (rs1387153) [OR: 1.44, 95\% CI: 1.01-2.08; $p=$ 0.047 (recessive model)]. Interestingly, after Bonferroni correction $(p<0.006$ indicates statistical significance), SNP rs13266634 of the SLC30A8 gene remains significantly associated with T2D susceptibility (OR: 1.81, 95\% CI: 1.31-2.50; $\mathrm{p}=0.0003$ ). No associations between CDKAL1 (rs7756992) or UBE2E2 (rs7612463) and T2D were observed in any of the 3 genetic models (Table 2).

\section{Analysis of association between SNPs and quantitative traits}

The 5 SNPs that were found to be significantly associated with T2D were then evaluated for association with

Table 1 Demographic, anthropometric, and clinical characteristics of the control and patient study groups

\begin{tabular}{|c|c|c|c|}
\hline Clinical characteristics & Controls $(n=500)$ & Patients $(n=500)$ & $p$-value \\
\hline Gender (M, F) (\%) & $28.8,71.2$ & $32.8,67.2$ & $0.170164: 336$ \\
\hline Age (yrs) (years) & $53.0 \pm 8.4$ & $57.2 \pm 12.2$ & $<0.001^{a}$ \\
\hline Age at diagnosis (yrs) & - & $49.4 \pm 11.3$ & ND \\
\hline Weight (kg) & $60.0 \pm 10.0$ & $67.7 \pm 13.8$ & $<0.001^{a}$ \\
\hline Waist circumference $(\mathrm{cm})$ & $83.3 \pm 9.4$ & $89.6 \pm 11.4$ & $<0.001^{a}$ \\
\hline Waist-to-hip ratio & $0.8 \pm 0.1$ & $0.9 \pm 0.1$ & $<0.001^{a}$ \\
\hline $\mathrm{BMI}\left(\mathrm{kg} / \mathrm{m}^{2}\right)$ & $24.1 \pm 3.3$ & $27.3 \pm 5.0$ & $<0.001^{a}$ \\
\hline $\mathrm{SBP}(\mathrm{mmHg})$ & $116.7 \pm 15.4$ & $134.9 \pm 19.4$ & $<0.001^{a}$ \\
\hline $\mathrm{DBP}(\mathrm{mmHg})$ & $71.7 \pm 10.3$ & $79.3 \pm 10.4$ & $<0.001^{a}$ \\
\hline FPG $(\mathrm{mmol} / \mathrm{l})$ & $3.9(3.4,4.4)$ & $8.4(6.6,12.1)$ & $<0.001^{b}$ \\
\hline $\mathrm{HbA}_{1 \mathrm{c}}(\mathrm{mmol} / \mathrm{mol})$ & $36.6(34.2,37.7)$ & $57.4(48.0,74.9)$ & $<0.001$ \\
\hline TC (mmol/l) & $208.4 \pm 37.9$ & $200.2 \pm 53.3$ & $<0.001^{a}$ \\
\hline TG $(\mathrm{mmol} / \mathrm{l})$ & $2.3(2.0,2.6)$ & $1.5(1.1,2.1)$ & $<0.001^{b}$ \\
\hline LDL-C (mmol/l) & $2.5(1.8,3.5)$ & $2.1(1.2,3.6)$ & $<0.001^{b}$ \\
\hline $\mathrm{HDL}-\mathrm{C}(\mathrm{mmol} / \mathrm{l})$ & $3.2(2.6,3.9)$ & $1.6(1.1,2.2)$ & $<0.001^{b}$ \\
\hline
\end{tabular}

Data presented as mean \pm standard deviation or median and interquartile range

A $p$-value $<0.05$ indicates statistical significance

Gender distribution between patients and controls was analyzed using Pearson's $x 2$ test

Comparison of quantitative variables between patients and controls was performed using Student's t-test or Mann-Whitney $U$ test

${ }^{a} p$-values and ${ }^{b} p$-values were determined using Student's t-test and Mann-Whitney $U$ test, respectively

Abbreviations: $M$ male, $F$ female, $B M I$ body mass index, SBP systolic blood pressure, DBP diastolic blood pressure, $F P G$ fasting plasma glucose, $H b A_{1 c}$ glycated

hemoglobin, TC total cholesterol, TG triglycerides, LDL-C low-density lipoprotein cholesterol, HDL-C high-density lipoprotein cholesterol, ND not determined 
Table 2 Association between eight SNPs and T2D in Thai population

\begin{tabular}{|c|c|c|c|c|c|c|c|c|c|c|c|c|c|}
\hline \multirow[t]{3}{*}{ Gene } & \multirow{3}{*}{$\begin{array}{l}\text { SNP } \\
\text { (major/ } \\
\text { minor } \\
\text { alleles*) }^{*}\end{array}$} & \multirow{3}{*}{$\begin{array}{l}{ }^{\text {a Risk }} \\
\text { allele } \\
\text { freq. }\end{array}$} & \multirow{3}{*}{$\begin{array}{l}{ }^{\mathrm{b}} \text { Risk } \\
\text { allele } \\
\text { freq. }\end{array}$} & \multirow[t]{3}{*}{ Subject } & \multirow{2}{*}{\multicolumn{3}{|c|}{$\begin{array}{l}\text { Genotype frequency, n } \\
(\%)\end{array}$}} & \multicolumn{2}{|c|}{ Additive model } & \multicolumn{2}{|c|}{$\underline{\text { Dominant model }}$} & \multicolumn{2}{|c|}{ Recessive model } \\
\hline & & & & & & & & \multirow{2}{*}{$\begin{array}{l}P \\
\text { unadjusted } \\
\text { OR (95\% } \\
\text { Cl) }\end{array}$} & \multirow{2}{*}{$\begin{array}{l}P \text { adjusted } \\
\text { OR }(95 \% \\
\text { Cl) }\end{array}$} & \multirow{2}{*}{$\begin{array}{l}P \\
\text { unadjusted } \\
\text { OR (95\% } \\
\text { Cl) }\end{array}$} & \multirow{2}{*}{$\begin{array}{l}P \text { adjusted } \\
\text { OR }(95 \% \\
\text { CI) }\end{array}$} & \multirow{2}{*}{$\begin{array}{l}P \\
\text { unadjusted } \\
\text { OR }(95 \% \\
\mathrm{Cl})\end{array}$} & \multirow{2}{*}{$\begin{array}{l}P \text { adjusted } \\
\text { OR (95\% } \\
\text { Cl) }\end{array}$} \\
\hline & & & & & A/A & $A / B$ & $\mathrm{~B} / \mathrm{B}$ & & & & & & \\
\hline \multirow[t]{3}{*}{ KCNQ1 } & \multirow{3}{*}{$\begin{array}{l}\text { rs2237892 } \\
(\underline{C}>\mathrm{T})\end{array}$} & \multirow[t]{3}{*}{0.76} & \multirow[t]{3}{*}{0.71} & Patient & 285 & 192 & 23 & \multirow{3}{*}{$\begin{array}{l}0.060 \\
1.74(0.99- \\
3.07)\end{array}$} & \multirow{3}{*}{$\begin{array}{l}0.020 \\
2.02(1.08- \\
3.79)\end{array}$} & \multirow{3}{*}{$\begin{array}{l}0.128 \\
1.53(0.88- \\
2.66)\end{array}$} & \multirow{3}{*}{$\begin{array}{l}0.057 \\
1.83(0.98- \\
3.42)\end{array}$} & \multirow{3}{*}{$\begin{array}{l}0.025 \\
1.36(1.04- \\
1.78)\end{array}$} & \multirow{3}{*}{$\begin{array}{l}0.018 \\
1.43(1.06- \\
1.92)\end{array}$} \\
\hline & & & & & $(57.0)$ & $(38.4)$ & $(4.6)$ & & & & & & \\
\hline & & & & Control & $\begin{array}{l}254 \\
(50.8)\end{array}$ & $\begin{array}{l}205 \\
(41.0)\end{array}$ & $\begin{array}{l}41 \\
(8.2)\end{array}$ & & & & & & \\
\hline \multirow[t]{2}{*}{$\begin{array}{l}\text { CDKN2A } \\
2 B\end{array}$} & \multirow[t]{2}{*}{$\begin{array}{l}\text { rs } 10811661 \\
(\underline{T}>C)\end{array}$} & \multirow[t]{2}{*}{0.68} & \multirow[t]{2}{*}{0.62} & Patient & $\begin{array}{l}228 \\
(45.6)\end{array}$ & $\begin{array}{l}220 \\
(44.0)\end{array}$ & $\begin{array}{l}52 \\
(10.4)\end{array}$ & \multirow{2}{*}{$\begin{array}{l}0.100 \\
1.45(0.93- \\
2.27)\end{array}$} & \multirow{2}{*}{$\begin{array}{l}0.044 \\
1.65(1.01- \\
2.71)\end{array}$} & \multirow{2}{*}{$\begin{array}{l}0.227 \\
1.29(0.85- \\
1.96)\end{array}$} & $\begin{array}{l}0.105 \\
1.46(0.92-\end{array}$ & $\begin{array}{l}0.077 \\
1.28(0.97-\end{array}$ & $\begin{array}{l}0.066 \\
1.33(0.98-\end{array}$ \\
\hline & & & & Control & $\begin{array}{l}185 \\
(37.0)\end{array}$ & $\begin{array}{l}248 \\
(49.6)\end{array}$ & $\begin{array}{l}67 \\
(13.4)\end{array}$ & & & & & & \\
\hline$S L C 30 A 8^{C}$ & $\begin{array}{l}\text { rs } 13266634 \\
(\underline{C}>\mathrm{T})\end{array}$ & 0.59 & 0.54 & Patient & $\begin{array}{l}172 \\
(34.4)\end{array}$ & $\begin{array}{l}242 \\
(48.4)\end{array}$ & $\begin{array}{l}86 \\
(17.2)\end{array}$ & $\begin{array}{l}0.040 \\
1.51(1.01-\end{array}$ & $\begin{array}{l}0.006 \\
1.86(1.19-\end{array}$ & $\begin{array}{l}0.309 \\
1.20(0.84-\end{array}$ & $\begin{array}{l}0.191 \\
1.29(0.87-\end{array}$ & $\begin{array}{l}0.008 \\
1.48(1.11-\end{array}$ & $\begin{array}{l}0.0003 \\
1.81(1.31-\end{array}$ \\
\hline & & & & Control & $\begin{array}{l}137 \\
(27.4)\end{array}$ & $\begin{array}{l}268 \\
(53.6)\end{array}$ & $\begin{array}{l}95 \\
(19.0)\end{array}$ & & & & & & \\
\hline HHEX & $\begin{array}{l}r s 1111875 \\
(A>G)\end{array}$ & 0.33 & 0.29 & Patient & $\begin{array}{l}234 \\
(46.8)\end{array}$ & $\begin{array}{l}200 \\
(40.0)\end{array}$ & $\begin{array}{l}66 \\
(13.2)\end{array}$ & $\begin{array}{l}0.039 \\
1.61(1.02-\end{array}$ & $\begin{array}{l}0.082 \\
1.55(0.94-\end{array}$ & $\begin{array}{l}0.260 \\
1.16(0.89-\end{array}$ & $\begin{array}{l}0.367 \\
1.14(0.85-\end{array}$ & $\begin{array}{l}0.044 \\
1.56(1.01-\end{array}$ & $\begin{array}{l}0.087 \\
1.51(0.94-\end{array}$ \\
\hline & & & & Control & $\begin{array}{l}254 \\
(50.8)\end{array}$ & $\begin{array}{l}201 \\
(40.2)\end{array}$ & $\begin{array}{l}45 \\
(9.0)\end{array}$ & & & & & & \\
\hline CDKAL1 & $\begin{array}{l}r s 7756992 \\
(A>\underline{G})\end{array}$ & 0.43 & 0.40 & Patient & $\begin{array}{l}156 \\
(31.2)\end{array}$ & $\begin{array}{l}257 \\
(51.4)\end{array}$ & $\begin{array}{l}87 \\
(17.4)\end{array}$ & $\begin{array}{l}0.249 \\
1.27(0.84-\end{array}$ & $\begin{array}{l}0.107 \\
1.44(0.92-\end{array}$ & $\begin{array}{l}0.355 \\
1.14(0.85-\end{array}$ & $\begin{array}{l}0.169 \\
1.25(0.90-\end{array}$ & $\begin{array}{l}0.340 \\
1.19(0.83-\end{array}$ & $\begin{array}{l}0.182 \\
1.30(0.88-\end{array}$ \\
\hline & & & & Control & $\begin{array}{l}171 \\
(34.2)\end{array}$ & $\begin{array}{l}254 \\
(50.8)\end{array}$ & $\begin{array}{l}75 \\
(15.0)\end{array}$ & & & & & & \\
\hline TCF7L2 & $\begin{array}{l}\text { rs } 7903146 \\
(C>I)\end{array}$ & 0.08 & 0.04 & Patient & $\begin{array}{l}429 \\
(85.8)\end{array}$ & $\begin{array}{l}67 \\
(13.4)\end{array}$ & $4(0.0)$ & NA & NA & $\begin{array}{l}0.006 \\
1.80(1.18-\end{array}$ & $\begin{array}{l}0.025 \\
1.70(1.06-\end{array}$ & NA & NA \\
\hline & & & & Control & $\begin{array}{l}456 \\
(91.2)\end{array}$ & $\begin{array}{l}44 \\
(8.8)\end{array}$ & $0(0.0)$ & & & & & & \\
\hline MTNR1B & $\begin{array}{l}r s 1387153 \\
(C>I)\end{array}$ & 0.44 & 0.42 & Patient & $\begin{array}{l}164 \\
(32.7)\end{array}$ & $\begin{array}{l}232 \\
(46.3)\end{array}$ & $\begin{array}{l}104 \\
(21.0)\end{array}$ & $\begin{array}{l}0.113 \\
1.35(0.93-\end{array}$ & $\begin{array}{l}0.120 \\
1.38(0.91-\end{array}$ & $\begin{array}{l}0.618 \\
1.07(0.80-\end{array}$ & $\begin{array}{l}0.757 \\
1.05(0.76-\end{array}$ & $\begin{array}{l}0.058 \\
1.38(0.98-\end{array}$ & $\begin{array}{l}0.047 \\
1.44(1.01-\end{array}$ \\
\hline & & & & Control & $\begin{array}{l}174 \\
(34.8)\end{array}$ & $\begin{array}{l}231 \\
(46.2)\end{array}$ & $\begin{array}{l}95 \\
(19.0)\end{array}$ & & & & & & \\
\hline UBE2E2 & $\begin{array}{l}\text { rs7612463 } \\
(\underline{C}>\mathrm{A})\end{array}$ & 0.83 & 0.82 & Patient & $\begin{array}{l}346 \\
(69.3)\end{array}$ & $\begin{array}{l}134 \\
(26.6)\end{array}$ & $\begin{array}{l}20 \\
(4.0)\end{array}$ & $\begin{array}{l}0.875 \\
0.94(0.47-\end{array}$ & $\begin{array}{l}0.855 \\
0.93(0.42-\end{array}$ & $\begin{array}{l}0.890 \\
0.95(0.47-\end{array}$ & $\begin{array}{l}0.872 \\
0.93(0.43-\end{array}$ & $\begin{array}{l}0.847 \\
0.97(0.72-\end{array}$ & $\begin{array}{l}0.826 \\
0.96(0.70-\end{array}$ \\
\hline & & & & Control & $\begin{array}{l}339 \\
(67.8)\end{array}$ & $\begin{array}{l}141 \\
(28.2)\end{array}$ & $\begin{array}{l}20 \\
(4.0)\end{array}$ & 1. & & & & & \\
\hline
\end{tabular}

Abbreviations: SNP single nucleotide polymorphism, $T 2 D$ type 2 diabetes, Freqfrequency, $A / A$ homo major allele, $A / B$ heterozygote allele, $B / B$ homo minor allele, $O R$ odds ratio, $\mathrm{Cl}$ confidence interval, $\mathrm{NA}$ not applicable (analytical model not applicable due to low frequency)

A $\mathrm{p}<0.006$ ( 0.05 divided by 8 , the total number of SNPs studied) was regarded as being statistically significant

$P$-values were calculated for the additive, dominant, and recessive genetic models using logistic regression with/without adjustment for age, gender, and body mass index. The OR and $95 \% \mathrm{Cl}$ of having the risk allele are shown

*Alleles in bold are the risk alleles for T2D identified in previous studies, while underlined alleles indicate the risk alleles for T2D observed in this study

${ }^{\mathrm{a}}$ Risk allele frequency in patients; ${ }^{\mathrm{b}}$ Risk allele frequency in controls; ${ }^{\mathrm{C}}$ Statistically significant after Bonferroni correction (multiple-testing) with $p$-value $<0.006$

quantitative traits, including body weight, waist circumference, WHR, BMI, FPG, $\mathrm{HbA}_{1 \mathrm{c}}$, TC, TG, LDL-C, and HDL-C using linear regression analysis after adjusting for age, sex, BMI, and medication (as appropriate) in both cases and controls (Table 3). Our analysis revealed $C D K N 2 A / 2 B$ (rs10811661) to be significantly associated with both waist circumference and WHR $(p=0.007$ and $p=0.023$, respectively). TCF7L2 (rs7903146) was associated with HDL-C $(p=0.023)$, and SLC3OA8 (rs13266634) was associated with $\mathrm{HbA}_{1 \mathrm{c}}(p=0.018)$. KCNQ1 (rs2237892) was significantly associated at borderline with both FPG and $\mathrm{HbA}_{1 \mathrm{c}}(p=0.050$ and $p=0.052$, respectively). No significant associations were observed between any SNPs and any quantitative traits in control subjects (data not shown).

\section{Discussion}

The present study evaluated 8 SNPs in or near the KCNQ1, CDKN2A/2B, SLC30A8, HHEX, CDKAL1, TCF7L2, MTNR1B, and UBE2E2 genes for significant association with T2D. Of those, 5 SNPs [KCNQ1 (rs2237892), CDKN2A/2B (rs10811661), SLC30A8 (rs13266634), TCF7L2 (rs7903146) and MTNR1B (rs1387153)] were found to be associated with T2D in 
Table 3 Association between CDKN2A/2B (rs10811661), SLC30A8 (rs13266634), and TCF7L2 (rs7903146) genotypes and quantitative traits among Thai patients with T2D

\begin{tabular}{|c|c|c|c|c|c|c|c|c|c|c|c|}
\hline \multirow{2}{*}{$\begin{array}{l}\text { Clinical } \\
\text { characteristics }\end{array}$} & \multicolumn{4}{|c|}{ CDKN2A/2B (rs10811661) genotypes } & \multicolumn{4}{|c|}{ SLC30A8 (rs13266634) genotypes } & \multicolumn{3}{|c|}{ TCF7L2 (rs7903146) genotypes } \\
\hline & $\overline{\mathrm{T} / \mathrm{T}}$ & $\mathrm{T} / \mathrm{C}$ & $\mathrm{C} / \mathrm{C}$ & $P$ & $\mathrm{C} / \mathrm{C}$ & $\mathrm{C} / \mathrm{T}$ & $\mathrm{T} / \mathrm{T}$ & $P$ & $\mathrm{C} / \mathrm{C}$ & $\mathrm{C} / \mathrm{T}+\mathrm{TT}$ & $P$ \\
\hline $\begin{array}{l}\text { umber of } \\
\text { atients }\end{array}$ & 228 & 220 & 52 & - & 172 & 242 & 86 & - & 429 & 71 & - \\
\hline Veight (kg) & $\begin{array}{l}68.99 \pm \\
13.96\end{array}$ & $\begin{array}{l}67.42 \pm \\
13.73\end{array}$ & $\begin{array}{l}65.01 \pm \\
14.28\end{array}$ & 0.250 & $\begin{array}{l}66.38 \pm \\
14.78\end{array}$ & $\begin{array}{l}66.38 \pm \\
14.78\end{array}$ & $\begin{array}{l}66.38 \pm \\
14.78\end{array}$ & 0.867 & $\begin{array}{l}67.58 \pm \\
13.60\end{array}$ & $\begin{array}{l}68.83 \pm \\
15.45\end{array}$ & 0.17 \\
\hline $\begin{array}{l}\text { Waist } \\
\text { circumference }(\mathrm{cm})\end{array}$ & $\begin{array}{l}89.34 \pm \\
11.32\end{array}$ & $\begin{array}{l}89.39 \pm \\
11.69\end{array}$ & $\begin{array}{l}92.59 \pm \\
11.04\end{array}$ & 0.007 & $\begin{array}{l}89.55 \pm \\
11.58\end{array}$ & $\begin{array}{l}89.79 \pm \\
10.82\end{array}$ & $\begin{array}{l}89.87 \pm \\
12.75\end{array}$ & 0.506 & $\begin{array}{l}89.66 \pm \\
11.30\end{array}$ & $\begin{array}{l}89.84 \pm \\
12.27\end{array}$ & 0.86 \\
\hline Waist-to-hip ratio & $0.89 \pm 0.07$ & $0.91 \pm 0.08$ & $0.92 \pm 0.11$ & 0.023 & $0.91 \pm 0.07$ & $0.90 \pm 0.08$ & $0.90 \pm 0.08$ & 0.263 & $0.90 \pm 0.08$ & $0.90 \pm 0.080$ & 0.3 \\
\hline BMI $\left(\mathrm{kg} / \mathrm{m}^{2}\right)$ & $27.60 \pm 5.06$ & $\begin{array}{l}27.07 \pm \\
4.99\end{array}$ & $\begin{array}{l}26.82 \pm \\
5.075\end{array}$ & 0.442 & $\begin{array}{l}26.65 \pm \\
5.15\end{array}$ & $\begin{array}{l}27.62 \pm \\
4.72\end{array}$ & $27.59 \pm 5.38$ & 0.273 & $\begin{array}{l}27.27 \pm \\
5.067\end{array}$ & $27.14 \pm 4.71$ & 0.7 \\
\hline SBP ( & $\begin{array}{l}133.60 \pm \\
19.99\end{array}$ & $\begin{array}{l}134.21 \pm \\
17.58\end{array}$ & $\begin{array}{l}143.52 \pm \\
23.29\end{array}$ & 0.746 & $\begin{array}{l}134.94 \pm \\
18.56\end{array}$ & $\begin{array}{l}134.44 \pm \\
19.30\end{array}$ & $\begin{array}{l}136.47 \pm \\
21.82\end{array}$ & 0.740 & $\begin{array}{l}135.25 \pm \\
19.64\end{array}$ & $\begin{array}{l}132.98 \pm \\
18.15\end{array}$ & 0.80 \\
\hline $\mathrm{DBP}(\mathrm{mmHg})$ & $\begin{array}{l}78.84 \pm \\
10.72\end{array}$ & $\begin{array}{l}79.42 \pm \\
9.87\end{array}$ & $\begin{array}{l}80.75 \pm \\
11.65\end{array}$ & 0.228 & $\begin{array}{l}79.95 \pm \\
10.16\end{array}$ & $\begin{array}{l}78.56 \pm \\
10.85\end{array}$ & $80.18 \pm 9.89$ & 0.348 & $\begin{array}{l}79.43 \pm \\
10.62\end{array}$ & $78.87 \pm 9.21$ & 0.4 \\
\hline FPG $(\mathrm{mmol} / \mathrm{l})$ & $\begin{array}{l}9.27(7.05 \\
12.49)\end{array}$ & $\begin{array}{l}8.16(6.38 \\
11.88)\end{array}$ & $\begin{array}{l}7.79(6.33 \\
10.68)\end{array}$ & 0.244 & $\begin{array}{l}8.33(6.64 \\
11.62)\end{array}$ & $\begin{array}{l}8.49(6.38 \\
12.93)\end{array}$ & $\begin{array}{l}8.77(6.84 \\
11.94)\end{array}$ & 0.164 & $\begin{array}{l}8.49(6.50 \\
12.25)\end{array}$ & $\begin{array}{l}8.55(6.94 \\
8.55)\end{array}$ & 0.7 \\
\hline $\mathrm{HbA}_{1 \mathrm{c}}(\mathrm{mmol} / \mathrm{mol})$ & $\begin{array}{l}59.05(49.70 \\
78.92)\end{array}$ & $\begin{array}{l}56.30 \\
(48.6,71.6)\end{array}$ & $\begin{array}{l}53.00 \\
(44.82,77.3)\end{array}$ & 0.683 & $\begin{array}{l}56.30 \\
(48.6,71.6)\end{array}$ & $\begin{array}{l}57.40 \\
(49.7,76.5)\end{array}$ & $\begin{array}{l}59.05(48.32, \\
78.10)\end{array}$ & 0.018 & $\begin{array}{l}57.40 \\
(48.60,74.9)\end{array}$ & $\begin{array}{l}56.30(47.50 \\
56.30)\end{array}$ & 0.53 \\
\hline $\mathrm{TC}(\mathrm{mmol} / \mathrm{l})$ & $\begin{array}{l}202.96 \pm \\
53.19\end{array}$ & $\begin{array}{l}197.52 \pm \\
51.12\end{array}$ & $\begin{array}{l}195.80 \pm \\
60.28\end{array}$ & 0.748 & $\begin{array}{l}197.40 \pm \\
55.56\end{array}$ & $\begin{array}{l}199.65 \pm \\
51.20\end{array}$ & $\begin{array}{l}208.33 \pm \\
55.57\end{array}$ & 0.853 & $\begin{array}{l}200.65 \pm \\
54.08\end{array}$ & $\begin{array}{l}198.00 \pm \\
49.65\end{array}$ & 0.59 \\
\hline TG $(\mathrm{mmol} / \mathrm{l})$ & $\begin{array}{l}1.46(1.03 \\
2.06)\end{array}$ & $\begin{array}{l}1.50(1.18, \\
2.11)\end{array}$ & $\begin{array}{l}1.64(1.17 \\
2.06)\end{array}$ & 0.197 & $\begin{array}{l}1.43(1.02 \\
2.05)\end{array}$ & $\begin{array}{l}1.51(1.14 \\
2.08)\end{array}$ & $\begin{array}{l}1.51(1.18 \\
2.26)\end{array}$ & 0.664 & $\begin{array}{l}1.52(1.15 \\
2.10)\end{array}$ & $\begin{array}{l}1.32(1.06 \\
1.32)\end{array}$ & 0.6 \\
\hline LDL-C (mmol/l) & $\begin{array}{l}2.22(1.39 \\
3.85)\end{array}$ & $\begin{array}{l}2.15(1.30 \\
3.50)\end{array}$ & $\begin{array}{l}1.47(1.10 \\
2.87)\end{array}$ & 0.943 & $\begin{array}{l}1.89(1.34 \\
3.64)\end{array}$ & $\begin{array}{l}2.38(1.22, \\
3.78)\end{array}$ & $\begin{array}{l}2.22(1.37 \\
3.71)\end{array}$ & 0.854 & $\begin{array}{l}2.17(1.33, \\
3.68)\end{array}$ & $\begin{array}{l}1.71 \text { (1.16, } \\
1.71)\end{array}$ & 0.7 \\
\hline $\mathrm{HDL}-\mathrm{C}(\mathrm{mmol} / \mathrm{l})$ & $\begin{array}{l}1.58(1.14 \\
2.21)\end{array}$ & $\begin{array}{l}1.63(1.19 \\
2.265)\end{array}$ & $\begin{array}{l}1.78(1.44 \\
2.32)\end{array}$ & 0.965 & $\begin{array}{l}1.64(1.17 \\
2.24)\end{array}$ & $\begin{array}{l}1.61(1.19 \\
2.17)\end{array}$ & $\begin{array}{l}1.55(1.14 \\
2.50)\end{array}$ & 0.464 & $\begin{array}{l}1.60(1.16 \\
2.18)\end{array}$ & $\begin{array}{l}1.81(1.34 \\
1.81)\end{array}$ & 0.02 \\
\hline
\end{tabular}

Data presented as mean \pm standard deviation or median and interquartile range

$P$-values were calculated using linear regression after adjusting for age, gender, BMI, and medication, as appropriate. FPG, $\mathrm{HbA1c}$, TG, LDL-C, and HDL-C values were log-transformed to improve normality before regression analysis

Abbreviations: $T 2 D$ type 2 diabetes, $B M I$ body mass index, $S B P$ systolic blood pressure, $D B P$ diastolic blood pressure, $F P G$ fasting plasma glucose, $H b A_{1 c}$ glycated hemoglobin, $T C$, total cholesterol, $T G$, triglycerides, $H D L-C$ high-density lipoprotein cholesterol, $L D L-C$ low-density lipoprotein cholesterol

Thai population. We also found association between CDKN2A/2B (rs10811661), SLC30A8 (rs13266634), and TCF7L2 (rs7903146) and diabetes-related quantitative traits.

SLC30A8 (solute carrier family 30 member 8 ) encodes zinc transporter-8 (ZnT8), which is specifically expressed in pancreatic endocrine cells and may participate in insulin secretion. SLC $30 A 8$ is expressed at high levels only in the pancreas, and is highly expressed in insulin-secreting beta cells $[17,18]$. Association between SNP rs13266634 in SLC30A8 and T2D risk was discovered in European subjects $[7,9,10]$. The presence of risk $\mathrm{C}$ allele of rs13266634 (SLC30A8) was found to be associated with increased risk of developing T2D in multiethnic case-control studies, including Asians [19, 20] and Europeans $[21,22]$. This SNP also showed strong association with T2D in our study with an OR of 1.78 [95\% CI: 1.31$2.50 ; p=3.0 \times 10^{-4}$ (recessive model)], which is higher than the odds ratios reported in previous studies (OR range: $1.1-1.2)$. The risk $C$ allele frequency of $59.0 \%$ in our population was similar to frequencies reported in
Asian populations (Han Chinese: 54.4\%, Japanese: 52.4\%), but different from European (71.7\%) population (source: dbSNP database). We also found SLC30A8 (rs13266634) to be associated with $\mathrm{HbA}_{1 \mathrm{c}}(p=0.018)$. A similar finding was observed in a Chinese study population [23]. Functional study found that zinc transporter 8 (ZnT8) is required for normal insulin crystallization and insulin processing and secretion [24]. In addition, subjects who had homozygous of the risk C allele of SLC3OA8 showed decreased peripheral insulin levels in the early phase of intravenous glucose tolerance test (GTT) [22]. This suggests that SLC30A8 may influence the $\mathrm{Zn}$ transporter, thereby affecting insulin stability and insulin secretion, but the precise role of variants of this gene remains unclear.

TCF7L2 (Transcription Factor 7-Like 2) is a transcription factor protein that is capable of binding beta catenin, which is involved in Wnt receptor signaling. This signaling pathway affects the expression of several genes, including insulin and glucagon genes [25]. A strong association was identified between TCF7L2 genetic variants and risk of T2D in a European population [8], 
which was then replicated in East Asian populations, including Chinese and Japanese $[15,26]$. In this study, we found significant association between SNP rs7903146 and increased risk of T2D in the dominant model (OR: 1.80, 95\% CI: $1.18-2.76 ; p=0.006)$. In contrast to populations with European and African ancestries, the risk $\mathrm{T}$ allele of rs7903146 was rare in our Thai cohort, with a minor allele frequency (MAF) of 0.08. The TCF7L2 gene was reported to be associated with T2D, insulin sensitivity, and insulin resistance [25]. In addition, the SNP rs7903146 was reported to be a risk factor for various metabolic disorders involving glucose and lipoprotein homeostasis [27, 28]. A previous study by our team showed significant association between an SNP in TCF7L2 and age of onset of diabetes [29]. In that study, we found that patients who carried a minor allele of an SNP were diagnosed with T2D at an earlier age. The present study also found association between SNP rs7903146 and HDL-C among T2D patients. The specific mechanism of TCF7L2 (rs7903146) that resides in a noncoding region and that drives the development of T2D remains unclear; however, the possible effect of the TCF7L2 risk allele is via a defect in insulin secretion [25].

KCNQ1 (potassium voltage gated channel, KQT like subfamily, member 1) plays a key role in potassium channels that control insulin secretion [30]. Common variants (SNP rs2237892, rs2237895, and rs2237897) in KCNQ1 were identified in East Asian population $[6,13]$. The association of these SNPs with T2D was replicated in European, Mexican, and Asian populations [6, 13, 31, 32]. Of the three aforementioned SNPs, SNP rs2237892 appeared to have the strongest association with T2D [13]. In the present study, we also found SNP rs2237892 to be significantly associated with T2D [OR: 2.02, 95\% CI: $1.08-3.79 ; p=0.020$ (additive model)] and [OR: 1.43, 95\% CI: $1.06-1.92 ; p=0.018$ (recessive model)]. Other studies found variants in KCNQ1 to be associated with impaired fasting plasma glucose, $\beta$-cell function, and metabolic traits $[6,13,33]$. However, we did not find evidence of association between KCNQ1 and diabetic-related quantitative traits. The potential mechanism of KCNQ1 and the pathogenesis of T2D needs to be further explored.

SNP rs10811661 is located $125 \mathrm{k}$-bases upstream from the cyclin-dependent kinase inhibitor $2 \mathrm{~A} / 2 \mathrm{~B}$ (CDKN2A) $2 B)$ gene on chromosome 9p21. SNP rs10811661 was found to be associated with risk of T2D in multiple large GWA studies $[4,9,10,34]$. In this study, we also found this SNP to be significantly associated with increased risk of T2D [OR: $1.65,95 \%$ CI: $1.01-2.71 ; p=0.044$ (additive model)]. The risk $\mathrm{T}$ allele was found to be more common in T2D patients (MAF: 0.68), which is similar to several previous reports in Caucasian and Asian populations [19, 35-37]. In addition, the risk allele of SNP rs10811661 was associated with both waist circumference and WHR among T2D patients in this study ( $p=$ 0.007 and $p=0.032$, respectively). In a similar result, a recent study from Vietnam found association between SNP rs10811661 and WHR in prediabetes subjects [38]. However, the mechanism by which the CDKN2A/2B locus influences diabetes risk remains uncertain. Recent publication showed $C D K N 2 A / 2 B$ locus SNPs may impact T2D risk by modulating islet gene expression and beta cell proliferation [39].

Several susceptibility genes have been identified and replicated for association with $\mathrm{T} 2 \mathrm{D}$ and diabetes-related traits in European and East Asians populations. Most of the susceptibility risk loci identified were shared among East Asians and populations of Europeans ancestry. However, a significant association signals at these shared loci seems to be independent among populations. This suggests that pathogenesis of the disease is common among populations, but that some of the risk variants may be population specific. Differences in genetic background, risk allele frequencies, and clinical characteristics, such as BMI cut-point for metabolic syndrome, diet, culture, and other lifestyle factors, may explain differences in risk variants between European and East Asians. It is, therefore, important to have information about the association of genetic variations and T2D, and to understand the roles of these T2D risk loci in different Asian populations, including Thai population. For example, rare PAX4 mutations were first identified in Thai MODY probands (designated MODY9) [40], but they are seldom found in those of European descent. A GWA study identified a susceptibility locus for T2D at 7q32 near PAX4 [41], and exome-chip association analysis revealed an Asian-specific missense variant in PAX4 associated with T2D in Chinese population [42]. Only one variant reached genome-wide significance $(P A X-\mathrm{Ar}$ g192His, rs2233580). This association is exclusive to East Asian individuals, in whom the $192 \mathrm{His}$ allele is common $(\mathrm{MAF}=10 \%)$ with a substantial effect size, with $192 \mathrm{His}$ observed to be virtually absent in individuals from other ancestries [43]. This suggests that PAX4 or T2D loci may be particularly relevant in the pathogenesis of T2D in both East Asians and in Thais.

With regard to the remaining 3 loci, HHEX (rs1111875), CDKAL1 (rs7756992), and UBE2E2 (rs7612463) showed no significant association with T2D in our cohort. This result may be explained by different environmental risk profiles between Europeans and Asians, different body composition and genetic backgrounds, different linkage disequilibrium patterns, or by the fact that we have insufficient statistical power with the current sample size to replicate some of these previously reported T2D risk loci. The sample size in this study yielded power to identify significant association between $\mathrm{T} 2 \mathrm{D}$ and variants in the 
KCNQ1, CDKN2A/2B, CDKAL1, HHEX, MTNR1B, TCF7L2, and UBE2E2 genes ranging from 2.13 to $37.41 \%$. A sample size ranging from 1094 to 12,600 would be needed to achieve an $80 \%$ power threshold to detect statistically significant results at alpha $=0.006$. The sample size included in this study for SLC3OA8 $(n=500)$ had $96 \%$ power to detect significant association with an OR of 1.81 assuming a minor allele frequency of 0.41 . This study has some mentionable limitations. First, the relatively small sample size and the commensurately limited statistical power that accompanied it may have limited our ability to identify all significant differences and associations. Second, several risk loci have been proposed as being associated with T2D in Caucasian and Asian populations. However, we limited our investigation to 8 SNPs from 8 candidate loci that were found to be common from studies in Caucasian and several different Asian populations. Thus, further studies that include the T2D risk loci not include in this study should be further investigated.

\section{Conclusion}

Of the eight genes included in our analysis, association was observed between KCNQ1, CDKN2A/2B, SLC30A8, TCF7L2, and MTNR1B loci and T2D in our Thai study population. Of these, $C D K N 2 A / 2 B, S L C 30 A 8$, and TCF7L2 genes were also associated with anthropometric, glycemic and lipid characteristics. Larger cohort studies and meta-analyses are needed to further confirm the effect of these variants in Thai population.

\section{Additional file}

Additional file 1: Table S1 Summary of the single nucleotide polymorphisms (SNPs) investigated in this study. Table S2 Primer sequence, PCR product size, and annealing temperature for HRM assay of designated SNPs. Table S3 Primer sequences of SLC30A8 (rs13266634) used for PCR-RFLP method. (DOCX 27 kb)

\section{Abbreviations}

ADA: American Diabetes Association; BMl: Body Mass index; bp: Base pair CDKAL 1: CDK5 regulatory subunit-associated protein 1-like 1; CDKN2A 2B: cyclin-dependent kinase inhibitor 2A; Cl: Confidence interval; DBP: diastolic blood pressure; FPG: fasting plasma glucose; GWA: genomewide association; $\mathrm{HbA}_{1}$ : glycated hemoglobin; HDL: High density lipoprotein; HDL-C: high-density lipoprotein; HHEX: hematopoieticallyexpressed homeobox; HWE: Hardy-Weinberg equilibrium; IDF: International Diabetes Federation; KCNQ1: potassium voltage-gated channel, KQT-like subfamily, member 1; LD: Linkage Disequilibrium; LDL: Low density lipoprotein; LDL-C: low-density lipoprotein; MAF: minor allele frequencies; MTNR1B: melatonin receptor type 1B; ND: Non-diabetic; OR: Odd ratio; RFLPPCR: Restriction fragment length - Polymerase Chain Reaction; SBP: systolic blood pressure; SLC30A8: zinc transporter member of solute carrier family 30; SNPS: single nucleotide polymorphisms; SPSS: Statistical Package for Social Science; T2D: type 2 diabetes; TC: Total cholesterol; TCF7L2: transcription factor-7-like 2; TG: Triglyceride; UBE2E2: Ubiquitin Conjugating Enzyme E2 E2; WHR: waist-to-hip ratio

\section{Acknowledgements}

The authors gratefully acknowledge the patients and control subjects that generously agreed to participate in this study. The authors would also like to thank Dr. Sirikul Kulanuwat for her helpful advice and guidance, and Mr. Choochai Nattuwakul for technical assistance.

\section{Funding}

This study was supported by a Siriraj Grant for Research Development and Medical Education, Mahidol University to NP, a grant from the Thailand Research Fund to PY (IRG5980006), a grant from the Thailand Research Fund Grant for New Researcher to WT (TRG5280002, TRG5780113), and a grant from the Faculty of Medicine Siriraj Hospital, Mahidol University to CC and PY. The funding body played no role in the design of the study, data collection, analysis, interpretation or writing of the manuscript.

\section{Availability of data and materials}

The data used and identified in this study are included within the article and in the provided supplementary data file.

\section{Authors' contributions}

$N P, C C, N C, P Y, M H$ and $W T$ contributed to the conceptualization of the study and obtained funding. NP and PY designed the study and interpreted data. CC and NJ performed the analyses. WT curated the data and oversaw laboratory work. All authors contributed to editing the manuscript, review and provided intellectual input to the final manuscript. All authors read and approved the final manuscript.

Ethics approval and consent to participate

Informed written consent was obtained from all the patients involved in this study. This study was approved by the Siriraj Institutional Review Board (SIRB), Faculty of Medicine Siriraj Hospital, Mahidol University (COA no. Si491/2014).

\section{Consent for publication}

The patients participating in this study signed their consent for the publication of the results obtained. This statement was approved by the Siriraj Institutional Review Board (SIRB), Faculty of Medicine Siriraj Hospital, Mahidol University.

\section{Competing interests}

The authors declare that they have no competing interests.

\section{Publisher's Note}

Springer Nature remains neutral with regard to jurisdictional claims in published maps and institutional affiliations.

\section{Author details}

${ }^{1}$ Division of Endocrinology and Metabolism, Department of Medicine, Faculty of Medicine Siriraj Hospital, Mahidol University, Bangkok, Thailand. ${ }^{2}$ Research Division, Department of Research and Development, Faculty of Medicine Siriraj Hospital, Mahidol University, Bangkok, Thailand. ${ }^{3}$ Department of Immunology, Faculty of Medicine Siriraj Hospital, Mahidol University, Bangkok, Thailand. ${ }^{4}$ Siriraj Center of Research Excellence for Molecular Medicine, Department of Research and Development, Faculty of Medicine Siriraj Hospital Mahidol University, Bangkok, Thailand. ${ }^{5}$ Department of Preventive and Social Medicine, Faculty of Medicine Siriraj Hospital, Mahidol University, Bangkok, Thailand.

Received: 7 August 2017 Accepted: 23 May 2018

Published online: 05 June 2018

\section{References}

1. Cho NH, Shaw JE, Karuranga S, Huang Y, da Rocha Fernandes JD, Ohlrogge AW, et al. IDF diabetes atlas: global estimates of diabetes prevalence for 2017 and projections for 2045. Diabetes Res Clin Pract. 2018;

2. Kahn SE, Cooper ME, Del Prato S. Pathophysiology and treatment of type 2 diabetes: perspectives on the past, present, and future. Lancet. 2014; 383(9922):1068-83.

3. Expert Committee on the Diagnosis and Classification of Diabetes Mellitus. Report of the expert committee on the diagnosis and classification of diabetes mellitus. Diabetes Care. 2003;26 Suppl 1:S5-20.

4. Voight BF, Scott $L$, Steinthorsdottir V, Morris AP, Dina C, Welch RP, et al Twelve type 2 diabetes susceptibility loci identified through large-scale association analysis. Nat Genet. 2010;42(7):579-89. 
5. Yamauchi T, Hara K, Maeda S, Yasuda K, Takahashi A, Horikoshi M, et al. A genome-wide association study in the Japanese population identifies susceptibility loci for type 2 diabetes at UBE2E2 and C2CD4A-C2CD4B. Nat Genet. 2010:42(10):864-8.

6. Unoki H, Takahashi A, Kawaguchi T, Hara K, Horikoshi M, Andersen G, et al. SNPs in KCNQ1 are associated with susceptibility to type 2 diabetes in east Asian and European populations. Nat Genet. 2008;40(9):1098-102.

7. Sladek R, Rocheleau G, Rung J, Dina C, Shen L, Serre D, et al. A genomewide association study identifies novel risk loci for type 2 diabetes. Nature. 2007:445(7130):881-5.

8. Grant SF, Thorleifsson G, Reynisdottir I, Benediktsson R, Manolescu A, Sainz J, et al. Variant of transcription factor 7-like 2 (TCF7L2) gene confers risk of type 2 diabetes. Nat Genet. 2006;38(3):320-3.

9. Scott LJ, Mohlke KL, Bonnycastle LL, Willer CJ, Li Y, Duren WL, et al. A genome-wide association study of type 2 diabetes in Finns detects multiple susceptibility variants. Science. 2007;316(5829):1341-5.

10. Saxena R, Voight BF, Lyssenko V, Burtt NP, de Bakker PI, Chen H, et al. Genome-wide association analysis identifies loci for type 2 diabetes and triglyceride levels. Science. 2007;316(5829):1331-6.

11. Zeggini E, Weedon MN, Lindgren CM, Frayling TM, Elliott KS, Lango H, et al. Replication of genome-wide association signals in UK samples reveals risk loci for type 2 diabetes. Science. 2007;316(5829):1336-41.

12. Prasad RB, Groop L. Genetics of type 2 diabetes-pitfalls and possibilities. Genes (Basel). 2015;6(1):87-123.

13. Yasuda K, Miyake K, Horikawa Y, Hara K, Osawa H, Furuta H, et al. Variants in KCNQ1 are associated with susceptibility to type 2 diabetes mellitus. Nat Genet. 2008:40(9):1092-7.

14. Shu XO, Long J, Cai Q, Qi L, Xiang YB, Cho YS, et al. Identification of new genetic risk variants for type 2 diabetes. PLoS Genet. 2010;6(9):e1001127.

15. Hayashi T, Iwamoto Y, Kaku K, Hirose H, Maeda S. Replication study for the association of TCF7L2 with susceptibility to type 2 diabetes in a Japanese population. Diabetologia. 2007;50(5):980-4.

16. Whiting DR, Guariguata L, Weil C, Shaw J. IDF diabetes atlas: global estimates of the prevalence of diabetes for 2011 and 2030. Diabetes Res Clin Pract. 94(3):311-21.

17. Chimienti F, Favier A, Seve M. ZnT-8, a pancreatic beta-cell-specific zinc transporter. Biometals. 2005;18(4):313-7.

18. Kawasaki E. ZnT8 and type 1 diabetes. Endocr J. 59(7):531-7.

19. Lee $\mathrm{YH}$, Kang ES, Kim SH, Han SJ, Kim CH, Kim HJ, et al. Association between polymorphisms in SLC30A8, HHEX, CDKN2A/B, IGF2BP2, FTO, WFS1, CDKAL1, KCNQ1 and type 2 diabetes in the Korean population. J Hum Genet. 2008;53(11-12):991-8.

20. Omori S, Tanaka Y, Takahashi A, Hirose H, Kashiwagi A, Kaku K, et al. Association of CDKAL1, IGF2BP2, CDKN2A/B, HHEX, SLC30A8, and KCNJ11 with susceptibility to type 2 diabetes in a Japanese population. Diabetes. 2008;57(3):791-5

21. Saxena R, Elbers CC, Guo Y, Peter I, Gaunt TR, Mega JL, et al. Large-scale gene-centric meta-analysis across 39 studies identifies type 2 diabetes loci. Am J Hum Genet. 2012;90(3):410-25.

22. Boesgaard TW, Zilinskaite J, Vanttinen M, Laakso M, Jansson PA, Hammarstedt A, et al. The common SLC30A8 Arg325Trp variant is associated with reduced first-phase insulin release in 846 non-diabetic offspring of type 2 diabetes patients-the EUGENE2 study. Diabetologia. 2008;51(5):816-20.

23. Wei FJ, Cai CY, Yu P, Lv J, Ling C, Shi WT, et al. Quantitative candidate gene association studies of metabolic traits in Han Chinese type 2 diabetes patients. Genet Mol Res. 14(4):15471-81.

24. Nicolson TJ, Bellomo EA, Wijesekara N, Loder MK, Baldwin JM, Gyulkhandanyan AV, et al. Insulin storage and glucose homeostasis in mice null for the granule zinc transporter ZnT8 and studies of the type 2 diabetes-associated variants. Diabetes. 2009;58(9):2070-83.

25. Gloyn AL, Braun M, Rorsman P. Type 2 diabetes susceptibility gene TCF $7 L 2$ and its role in beta-cell function. Diabetes. 2009:58(4):800-2.

26. Wen J, Ronn T, Olsson A, Yang Z, Lu B, Du Y, et al. Investigation of type 2 diabetes risk alleles support CDKN2A/B, CDKAL1, and TCF7L2 as susceptibility genes in a Han Chinese cohort. PLoS One. 5(2):e9153.

27. Povel CM, Boer JM, Reiling E, Feskens EJ. Genetic variants and the metabolic syndrome: a systematic review. Obes Rev. 2011;12(11):952-67.

28. Perez-Martinez P, Perez-Caballero Al, Garcia-Rios A, Yubero-Serrano EM, Camargo A, Gomez-Luna MJ, et al. Effects of rs7903146 variation in the $\mathrm{Tcf} 7 \mathrm{I} 2$ gene in the lipid metabolism of three different populations. PLoS One. 2012;7(8):e43390.
29. Tangjittipokin W, Chongjarean N, Plengvidhya N, Homsanit M, Yenchitsomanus PT. Transcription factor 7-like 2 (TCF7L2) variations associated with earlier age-onset of type 2 diabetes in Thai patients. J Genet. 2012;91(2):251-5.

30. Jespersen T, Grunnet M, Olesen SP. The KCNQ1 potassium channel: from gene to physiological function. Physiology (Bethesda). 2005;20:408-16.

31. Hu C, Wang C, Zhang R, Ma X, Wang J, Lu J, et al. Variations in KCNQ1 are associated with type 2 diabetes and beta cell function in a Chinese population. Diabetologia. 2009;52(7):1322-5.

32. Been LF, Ralhan S, Wander GS, Mehra NK, Singh J, Mulvihill JJ, et al. Variants in KCNQ1 increase type II diabetes susceptibility in south Asians: a study of 3,310 subjects from India and the US. BMC Med Genet. 2011:12:18.

33. Chen Z, Yin Q, Ma G, Qian Q. KCNQ1 gene polymorphisms are associated with lipid parameters in a Chinese Han population. Cardiovasc Diabetol. 2010;9:35.

34. Steinthorsdottir $V$, Thorleifsson G, Reynisdottir I, Benediktsson R, Jonsdottir T, Walters $\mathrm{GB}$, et al. A variant in CDKAL1 influences insulin response and risk of type 2 diabetes. Nat Genet. 2007;39(6):770-5.

35. Sakai K, Imamura M, Tanaka Y, Iwata M, Hirose H, Kaku K, Maegawa H, Watada H, Tobe K, Kashiwagi A, Kawamori R, Maeda S. Replication study for the association of 9 East Asian GWAS-derived loci with susceptibility to type 2 diabetes in a Japanese population. PLoS One. 2013;8(9):e76317.

36. Grarup N, Rose CS, Andersson EA, Andersen G, Nielsen AL, Albrechtsen A, et al. Studies of association of variants near the HHEX, CDKN2A/B, and IGF2BP2 genes with type 2 diabetes and impaired insulin release in 10,705 Danish subjects: validation and extension of genome-wide association studies. Diabetes. 2007;56(12):3105-11.

37. Hu C, Zhang R, Wang C, Wang J, Ma X, Lu J, et al. PPARG, KCNJ11, CDKAL1, CDKN2A-CDKN2B, IDE-KIF11-HHEX, IGF2BP2 and SLC30A8 are associated with type 2 diabetes in a Chinese population. PLoS One. 2009;4(10):e7643.

38. Binh TQ, Thu NT, Phuong PT, Nhung BT, Nhung TT. CDKN2A-rs10811661 polymorphism, waist-hip ratio, systolic blood pressure, and dyslipidemia are the independent risk factors for prediabetes in a Vietnamese population. BMC Genet. 2015;16:107.

39. Kong Y, Sharma RB, Ly S, Stamateris RE, Jesdale WM, Alonso LC. CDKN2A/B T2D Genome-Wide Association Study Risk SNPs Impact Locus Gene Expression and Proliferation in Human Islets. Diabetes. 2018;67(5):872-84.

40. Plengvidhya N, Kooptiwut S, Songtawee N, Doi A, Furuta H, Nishi M, et al. PAX4 mutations in Thais with maturity onset diabetes of the young. J Clin Endocrinol Metab. 2007;92(7):2821-6.

41. Ma RC, Hu C, Tam CH, Zhang R, Kwan P, Leung TF, et al. Genome-wide association study in a Chinese population identifies a susceptibility locus for type 2 diabetes at 7q32 near PAX4. Diabetologia. 2013;56(6):1291-305.

42. Cheung CY, Tang CS, Xu A, Lee CH, Au KW, Xu L, et al. Exome-chip association analysis reveals an Asian-specific missense variant in PAX4 associated with type 2 diabetes in Chinese individuals. Diabetologia. 2017:60(1):107-15.

43. Fuchsberger C, Flannick J, Teslovich TM, Mahajan A, Agarwala V, Gaulton K, et al. The genetic architecture of type 2 diabetes. Nature. 2016;536(7614):41-7.

Ready to submit your research? Choose BMC and benefit from:

- fast, convenient online submission

- thorough peer review by experienced researchers in your field

- rapid publication on acceptance

- support for research data, including large and complex data types

- gold Open Access which fosters wider collaboration and increased citations

- maximum visibility for your research: over $100 \mathrm{M}$ website views per year

At BMC, research is always in progress.

Learn more biomedcentral.com/submissions 